\title{
The effect of pore size on permeability and cell attachment in collagen scaffolds for tissue engineering
}

\author{
Fergal J. O’Brien ${ }^{\mathrm{a}, \mathrm{b}, *}$, Brendan A. Harley, ${ }^{\mathrm{c}, \mathrm{d}}$, Mary A. Waller ${ }^{\mathrm{b}}$, Ioannis V. Yannas ${ }^{\mathrm{c}}$, \\ Lorna J. Gibson ${ }^{\mathrm{d}}$ and Patrick J. Prendergast ${ }^{\mathrm{b}}$ \\ ${ }^{a}$ Department of Anatomy, Royal College of Surgeons in Ireland, St Stephen's Green, Dublin, Ireland \\ ${ }^{\mathrm{b}}$ Trinity Centre for Bioengineering, Trinity College, Dublin, Ireland \\ ${ }^{\mathrm{c}}$ Department of Mechanical Engineering, Massachusetts Institute of Technology, Cambridge, MA, USA \\ ${ }^{\mathrm{d}}$ Department of Materials Science and Engineering, Massachusetts Institute of Technology, Cambridge, \\ MA, USA
}

\begin{abstract}
The permeability of scaffolds and other three-dimensional constructs used for tissue engineering applications is important as it controls the diffusion of nutrients in and waste out of the scaffold as well as influencing the pressure fields within the construct. The objective of this study was to characterize the permeability/fluid mobility of collagen-GAG scaffolds as a function of pore size and compressive strain using both experimental and mathematical modeling techniques. Scaffolds containing four distinct mean pore sizes $(151,121,110,96$ microns) were fabricated using a freeze-drying process. An experimental device was constructed to measure the permeability of the scaffold variants at different levels of compressive strain $(0,14,29$ and $40 \%)$ while a low-density open-cell foam cellular solids model utilizing a tetrakaidecahedral unit cell was used to accurately model the permeability of each scaffold variant at all level of applied strain. The results of both the experimental and the mathematical analysis revealed that scaffold permeability increases with increasing pore size and decreases with increasing compressive strain. The excellent comparison between experimentally measured and predicted scaffold permeability suggests that cellular solids modelling techniques can be utilized to predict scaffold permeability under a variety of physiological loading conditions as well as to predict the permeability of future scaffolds with a wide variety of pore microstructures.
\end{abstract}

Keywords: Collagen, scaffold, tissue engineering, permeability, cell attachment

\section{Introduction}

The goal of tissue engineering is to develop cell, construct, and living system technologies to restore the microstructure as well as functional mechanical properties of damaged or degenerated tissue. Tissue engineering relies extensively on the use of porous scaffolds to provide the appropriate microstructural, mechanical, and compositional environment to induce regeneration of injured tissues and organs. Scaffolds are typically seeded with cells and occasionally growth factors and are then either cultured in vitro to study cell behavior and tissue sythesis processes or are implanted in various anatomical sites

\footnotetext{
*Address for correspondence: Dr. Fergal J. O’Brien, Department of Anatomy, Royal College of Surgeons in Ireland, St. Stephens Green, Dublin 2, Ireland. Tel.: +353 1 4022149; Fax: +353 1 4022355; E-mail: fjobrien@rcsi.ie.
} 
to induce regeneration of damaged tissues or organs. In our laboratory, we use a composite collagenglycosaminoglycan (collagen-GAG, CG) scaffold as the basis for tissue engineered bone and cartilage. This scaffold is produced by a lyophilisation (freeze-drying) process that allows significant alteration of the scaffold pore microstructure by modifying the thermal processing conditions during fabrication.

The permeability of, and fluid mobility through porous materials are two intrinsic properties describing the relative ease that fluids can flow through cellular materials such as most tissue engineering scaffolds. Permeability $\left(k\right.$, units of $\left.\mathrm{m}^{2}\right)$ is the single-phase fluid conductivity of a porous material and is an intrinsic and quantitative parameter describing the scaffold structure independent of sample size and the fluid used. Scaffold permeability is defined by a combination of five important parameters: (1) porosity, (2) pore size and distribution, (3) pore interconnectivity (or tortuosity), (4) fenestration (pore interconnection) size and distribution, and (5) pore orientation. The fluid mobility ( $K$, units of $\mathrm{m}^{4} / \mathrm{Ns}$ ) of a scaffold is another intrinsic property defining fluid flow through a porous material and is defined as the material permeability normalized by the viscosity of the fluid $(\mu$, units of $\mathrm{Pa} \cdot \mathrm{s})$ :

$$
K=\frac{k}{\mu}
$$

In the tissue engineering literature describing characterization of a variety of biological materials and three-dimensional constructs such as gels and scaffolds, both the fluid mobility $(K)$ through and permeability $(k)$ of tissues and scaffolds are reported and are equally appropriate. While permeability and fluid mobility are often used interchangeably, the units of the reported values easily distinguish them. In order to quickly compare the mobility and permeability of many tissue engineering constructs and biological tissues, the viscosity of water can be used $(0.001 \mathrm{~Pa} \cdot \mathrm{s})$. For the remainder of this study we are reporting values of the fluid mobility (units $\mathrm{m}^{4} / \mathrm{Ns}$ ) of collagen-glycosaminoglycan scaffolds in order to describe its permeability characteristics.

As previously noted, the permeability of biological tissues and tissue-engineered scaffolds plays a significant role in the nutrient and waste transport within the structure. There are two mechanisms available for transport of metabolites to and waste products from cells in a scaffold: diffusion, and in the case of in vivo applications, transport through capillary networks formed throughout the scaffold via angiogenesis. While angiogenesis becomes the limiting factor in vivo, significant angiogenesis is not observed for the first few days after implantation, and is not present at all in vitro. Fluid flow, and therefore permeability, provides the means to transport nutrients to, and waste away from, cells. The influence of fluid flow has been studied in a number of tissues including bone [1], tumor tissue [2,3] and cartilage [4]. The permeability of articular cartilage is of interest because of the fluid mechanics of joint lubrication [4] and nutrient transport [5] of osteoarthritis. These studies have reported that the permeability of cartilage tissue ranges from 0.1 to $2 \times 10^{-15} \mathrm{~m}^{4} / \mathrm{Ns}\left(\sim 0.1\right.$ to $\left.2 \times 10^{-18} \mathrm{~m}^{2}\right)$ and that cartilage permeability is dependent on the composition, organisational depth [6], and the mechanical loading of the sample [4]. It has been shown that cartilage permeability decreases with increasing tissue depth as far as the calcified layer. This decrease has been correlated with an increase in proteoglycans and to a lesser extent an increase in collagen content. Scaffold and tissue permeability also influences cyclical changes in biophysical stimuli due to fluid flow through the structure during mechanical loading. Specifically, permeability affects the magnitude of pressure and fluid shear stresses within the construct or tissue, both of which have been identified as potential stimuli for cellular differentiation or functional adaptation [7-9]. Construct permeability has also been shown to influence the degradation rate of biodegradable scaffolds for tissue engineering [10].

As previously noted, the permeability of a scaffold is determined by a combination of microstructural factors including porosity, pore size, geometry, and distribution, pore interconnectivity, fenestration 
Table 1

Permeability of a variety of tissue engineering scaffolds as reported in the literature. Method 1 refers to direct measurement of construct permeability while method 2 refers to indirect measurement of construct permeability. The direct method applies a known pressure to the material, measures the flow rate, and calculates permeability according to Darcy's law. The indirect method applies mechanical load to the material, and curve-fits the reaction force vs. time curve to a theoretical model

\begin{tabular}{|c|c|c|c|c|c|c|}
\hline TE Scaffolds & Author & Material & Porosity & Method & Permeability & Units \\
\hline $\begin{array}{r}\text { Ceramic and } \\
\text { composites }\end{array}$ & $\begin{array}{l}\text { Shihong et al. (2003) } \\
\text { Haddock et al. (1999) }\end{array}$ & $\begin{array}{l}\text { Z-BCP; D-BCP; I-BCP } \\
\text { Coral; HA-CAM; HA-50 } \\
\text { Coralline hydroxyapatite }\end{array}$ & $\begin{array}{l}75 ; 74 ; 54 \\
- \\
-\end{array}$ & $\begin{array}{l}1 \\
1 \\
1\end{array}$ & $\begin{array}{l}0.01-0.35 \times 10^{-9} \\
0.05-0.35 \times 10^{-9} \\
1.7-8.6 \times 10^{-10}\end{array}$ & $\begin{array}{l}\mathrm{m}^{2} \\
\mathrm{~m}^{2} \\
\mathrm{~m}^{2}\end{array}$ \\
\hline Synthetic polymers & $\begin{array}{l}\text { Beatty et al. (2002) } \\
\text { Spain et al. (1998) } \\
\text { Agrawal et al. (2000) }\end{array}$ & $\begin{array}{l}\text { PGA-PLLA } \\
\text { PLA/PGA } \\
\text { PLA/PGA (salt:polymer) } \\
3.5-14: 1\end{array}$ & $\begin{array}{l}77 \\
51-71 \\
80-92\end{array}$ & $\begin{array}{l}2 \\
1 \\
1\end{array}$ & $\begin{array}{l}1.77 \pm 0.99 \times 10^{-15} \\
1.82 \pm 3.65 \times 10^{-9} \\
2-16.1 \times 10^{-9}\end{array}$ & $\begin{array}{l}\mathrm{m}^{4} / \mathrm{Ns} \\
\mathrm{m}^{4} / \mathrm{Ns} \\
\mathrm{m}^{4} / \mathrm{Ns}\end{array}$ \\
\hline Natural polymers & $\begin{array}{l}\text { Ramanujan et al. (2002) } \\
\text { Beatty et al. (2002) } \\
\text { Grattoni et al. (2001) }\end{array}$ & $\begin{array}{l}\text { Collagen gel } \\
\text { SIS } \\
\text { Polyacrylamide gel }\end{array}$ & $\begin{array}{l}1-4.5 \% \mathrm{w} / \mathrm{v} \\
- \\
1.8-3 \% \mathrm{w} / \mathrm{v}\end{array}$ & $\begin{array}{l}1 \\
2 \\
1\end{array}$ & $\begin{array}{l}1 \times 10^{-15}-10^{-16} \\
17.09 \pm 8.95 \times 10^{-15} \\
0.004-0.013 \times 10^{-12}\end{array}$ & $\begin{array}{c}\mathrm{m}^{2} \\
\mathrm{~m}^{4} / \mathrm{Ns} \\
\mathrm{m}^{2}\end{array}$ \\
\hline
\end{tabular}

size, and the orientation of pores with respect to flow direction [11]. Additionally, construct chemical composition can influence permeability. Different biological compounds such as glycosaminoglycans (e.g. hyaluronate or chondroitin sulfate) and collagen [12] have been shown to affect the permeability of many different tissues. In many polymer scaffold fabrication processes, salts are added to a polymerwater solution in order to remove water as charges in salt attract water molecules, and water molecules therefore bind to the salt instead of the polymer. The salt:polymer ratio of synthetic polymer scaffold systems has been shown to significantly affects its permeability [10]. Table 1 shows experimentally measured values of permeability of a series of different tissue engineering scaffolds. However, despite such previous research, very little data exists describing the permeability of natural polymer scaffolds. Ramanujan et al. [13] determined the permeability of type I collagen gels in the context of transport within tumor tissue. These collagen gels were prepared from $1-4.5 \%$ weight/volume solutions and polymerized by incubation; gel permeability ranged from $1 \times 10^{-15}$ to $10^{-16} \mathrm{~m}^{2}\left(\sim 0.1\right.$ to $\left.1 \times 10^{-12} \mathrm{~m}^{4} / \mathrm{Ns}\right)$. The permeability of a polyacrylamide (carbohydrate) gel (1.8-3\% weight/volume) has also been reported, ranging from 0.004 to $0.013 \times 10^{-12} \mathrm{~m}^{2}\left(\sim 0.4\right.$ to $\left.1.3 \times 10^{-11} \mathrm{~m}^{4} / \mathrm{Ns}\right)$ [14]. Beatty et al. [15] measured the permeability of small-intestinal submucosa (SIS), a decellularized, natural extracellur matrix derived from the intestinal lining of pigs that is used as a regeneration template for heart valves. SIS scaffold permeability, measured indirectly, was estimated to be $17 \times 10^{-15} \mathrm{~m}^{4} / \mathrm{Ns}\left(\sim 17 \times 10^{-18} \mathrm{~m}^{2}\right)$. Agrawal et al. [10] measured the permeability of 50:50 PLGA scaffold systems and found that permeability ranged from 2 to $16 \times 10^{-9} \mathrm{~m}^{4}$ /Ns $\left(\sim 2\right.$ to $\left.16 \times 10^{-12} \mathrm{~m}^{2}\right)$, and was dependent on the polymer:salt ratio. The permeability and porosity of these PLGA scaffolds was compared over time as the scaffold degraded; changes in permeability and porosity were not found to be similar suggesting a more substantial model of permeability is needed. Spain et al. [16] also characterized the permeability of PLGA constructs and reported similar values. While there are studies characterizing the permeability characteristics of specific synthetic and natural three-dimensional constructs and biological tissues, there is little in the literature to suggest a systematic framework to describe construct permeability. Additionally, the effects of compressive strain on scaffold permeability is an important feature to characterize because many tissue engineering constructs are utilized in load-bearing applications that can significantly influence both construct permeability and the resulting metabolite diffusion.

The objectives of this study were to characterise the permeability/fluid mobility of collagen-GAG (CG) scaffolds as a function of pore size and compressive strain, to investigate potential factors that influence construct permeability, to relate scaffold permeability data to previously observed changes in 
cellular adhesion within these scaffolds, and to develop a cellular solids model capable of quantitatively describing the permeability characteristics of a variety of scaffold microstructures under different loading conditions. Previously [17], we have studied the effects of pore size and structure of CG scaffolds on the attachment behaviour of osteoblast-like cells (MC3T3-E1 mouse clonal osteogenic cells). In that study, a homologous series of four scaffold variants with constant composition (CG) and solid volume fraction (0.005), but with four distinct pore sizes were utilized. With a constant solid volume fraction but variable pore size, these four scaffold variants had four distinct specific surface areas, defining the total surface of the scaffold available for cells to bind to. Cells were seeded onto the four scaffold variants and maintained in culture. The fraction of viable cells attached to the scaffold (compared to the total number of cells initially seeded) decreased with increasing average pore diameter, and increased linearly with scaffold specific surface area as calculated via a cellular solids model of the pore microstructure [17]. We hypothesized that the results of this previous study showed that the biological activity of three-dimensional constructs used for tissue engineering applications depends on the density of available ligands. Ligand density was hypothesized to be defined by the scaffold specific surface area, defining the total surface area of the scaffolds, and the scaffold chemical composition, which determines the type and surface density of specific ligands within the scaffold. Furthermore, we hypothesized that the permeability of these scaffolds would be inversely proportional to the specific surface area due to the frictional effects of fluid flow by the struts that define the scaffold microstructure. In this study, we aim to test this hypothesis by (i) characterizing the permeability and fluid mobility of the same series of collagen-GAG scaffolds, (ii) investigating the effect of pore size and compressive strain on scaffold permeability, (iii) using confocal reflection microscopy to provide microstructural information regarding changes in scaffold specific area due to changes in mean pore size, and (iv) developing a cellular solids model to mathematically describe construct permeability in terms of significant structural factors (e.g. mean pore size, applied strain, relative density).

\section{Material and methods}

\subsection{Scaffold fabrication}

The scaffolds used in this study were fabricated by a lyophilisation (freeze-drying) process. Recent work has led to the development of new processing techniques to control the rate of freezing during fabrication and the final pore microstructure [18]. The modified fabrication technique allows production of CG scaffolds with an equiaxed, uniform microstructure characterised by reduced variation in mean pore size throughout the scaffold compared to an original version of the scaffold [19] which received FDA approval in 1996 for use as dermal replacement in burns patients. Briefly, chondroitin 6-sulfate is coprecipitated with microfibrillar, type I collagen from bovine tendon in a solution of acetic acid. The aqueous CG suspension is then frozen, resulting in a continuous, interpenetrating network of ice crystals surrounded by the collagen-GAG co-precipitate. Sublimation removes the ice content, producing a highly porous sponge where the pores are defined by the size and shape of the individual ice crystals produced during solidification. While typical porosities of these scaffolds are above $99 \%$, the pore volume fraction and mean pore size can be controlled by modifying the volume fraction of the precipitate and the freezing temperature, respectively. By solidifying the CG suspension at a warmer freezing temperature, larger ice crystals form due to the reduced solidification rate and resultant increased time of ice crystal coarsening, resulting in scaffolds with increased mean pore size. In this study a homologous series of CG scaffolds were created using varied final freezing temperatures $\left(\mathrm{T}_{f}=-10,-20,-30,-40^{\circ} \mathrm{C}\right)$; this 
homologous series is characterized by a constant composition (CG) and solid volume fraction (0.005) but four distinct mean pore sizes $(151,121,110,96 \mu \mathrm{m})$ [17]. All CG scaffolds manufactured in this study were crosslinked via a standard dehydrothermal (DHT) process after freeze-drying to strengthen the collagen network by introducing covalent crosslinks between the polypeptide chains of the collagen fibers. Crosslinking was carried out in a vacuum oven (Fisher IsoTemp 201, Fisher Scientific, Boston, MA) at a temperature of $105^{\circ} \mathrm{C}$ under a vacuum of 50 mTorr for 24 hours [19]. This process also sterilised the scaffolds.

\subsection{Permeability analysis}

The permeability of the four scaffold variants was measured as a function of mean pore size and compressive strain using both (i) an experimental technique and (ii) mathematical modeling technique using a cellular solids modeling technique used previously to successfully describe the variation of CG scaffold specific surface area with mean pore size [17].

\subsubsection{Experimental (measured) permeability}

A device (Fig. 1) was constructed to measure the permeability of the scaffold variants under different levels of applied compressive strain. The device was constructed from two (top and bottom) brass plates, each with an attached $8 \mathrm{~mm}$ inner diameter tube. A stainless steel mesh was secured over the tube openings in both brass plates. The mesh, a medical-grade stainless steel (Braun, Frankfurt, Germany) was adhered to the brass plates with a high-grade cyanoacrylate glue (LocTite 401, Herts, UK). The mesh served a two-fold purpose: to physically support the scaffold above the tube opening in the bottom plate, and to apply compressive strain to the scaffold via the height-adjustable top plate. Stainless steel spacers of varying thickness $(2.1-5 \mathrm{~mm})$ were placed between the two stainless steel meshes when the testing rig was assembled, regulating the degree of applied compressive strain. Importantly, the mesh did not inhibit or disrupt fluid flow through the rig. To secure the scaffold in place, the scaffold edges (away from the tube openings) were clamped to the bottom stainless steel mesh using a silicone spacer which also served as a leak-proof seal for the rig, preventing pressure loss. The inner diameter of the silicone spacer $(10 \mathrm{~mm})$ was slightly larger than that of the brass plate $(8 \mathrm{~mm})$. Cylindrical scaffold disks, $13 \mathrm{~mm}$ in diameter and $3.5 \mathrm{~mm}$ thick, were cut from each scaffold variant using a dermal punch (Miltex, York, PA, USA) and then submerged in saline solution for 24 hours prior to testing in order to completely hydrate the scaffold.

Darcy's law states that permeability, $k$, can be calculated from the following expression (1) where $Q$ is the volumetric flow rate $(\mathrm{ml} / \mathrm{s}), \Delta P$ is the pressure difference across the sample $\left(\mathrm{N} / \mathrm{m}^{2}\right), l$ is length of the specimen through which the fluid flows (m), $A$ is the sample cross-sectional area in the direction of flow $\left(\mathrm{m}^{2}\right)$, and $\mu$ is the viscosity (Pa.s) of the fluid.

$$
k=\frac{Q \cdot l \cdot \mu}{\Delta P \cdot A}
$$

As previously noted, the degree of scaffold compression was controlled by the stainless steel spacers of $2.1,2.5,3$, and $5 \mathrm{~mm}$ thickness that fit around the screws between the top and bottom stainless steel meshes and brass plates. The thickness of the scaffolds was $3.5 \mathrm{~mm}$, making these spacers correspond to compressive strains of $40 \%, 29 \%, 14 \%$, and $0 \%$, respectively. The tube extending from the top brass plate was then attached to a reserve of saline solution pressurized with a $1.2 \mathrm{~m}$ saline solution pressure head. The flow rate of the saline solution through the scaffold was measured via the flow rate from the tube attached to the bottom plate of the permeability device using a stopwatch (Oregon Scientific, 


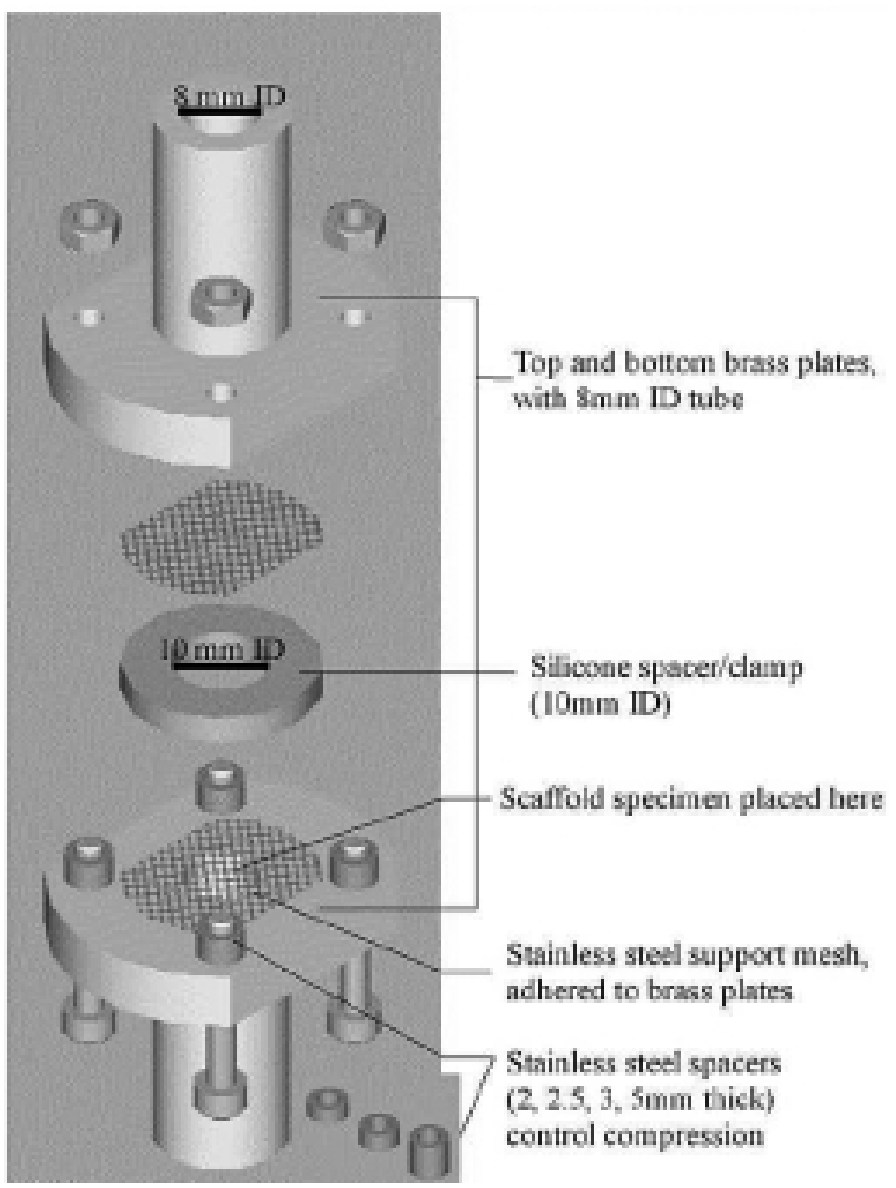

Fig. 1. The device utilized in this study to measure the permeability (fluid mobility) of collagen-GAG scaffolds. Stainless steel spacers of varying thickness regulated the degree of applied compressive strain while preventing fluid loss from the scaffold edges. The fours spacers of 5, 3, 2.5 and $2.1 \mathrm{~mm}$ give resultant strains of $0 \%, 14 \%, 29 \%$ and $40 \%$.

Portland, Oregon, USA) and an electronic scale accurate to within $0.05 \mathrm{~g}$ (Mettler Toledo, Greifensee, Switzerland) (Fig. 1).

The permeability $(k)$ and fluid mobility $(K)$ of each of the four scaffolds under each level of applied compressive strain was calculated using (2) a modified version of Equation 1 [16] where $Q$ was the measured flow rate $(\mathrm{ml} / \mathrm{s}), \mu$ is the saline solution viscosity, $r$ the radius of the scaffold sample $(4 \mathrm{~mm})$, $l$ the thickness of the sample, and $P$ the height of the saline solution column $(1.2 \mathrm{~m})$.

$$
\begin{aligned}
& k=\frac{324.8(Q \cdot l \cdot \mu)}{r^{2} P} \\
& K=\frac{k}{\mu}=\frac{324.8(Q \cdot l)}{r^{2} P}
\end{aligned}
$$

\subsubsection{Mathematical (calculated) permeability}

A low-density, open-cell foam cellular solids model utilising a tetrakaidecahedral unit was used to model the permeability of CG scaffolds with variable mean pore size under variable applied compressive 
strain. We have previously shown that cellular solids modeling techniques using a tetrakaidecahedral unit cell can accurately represent and predict salient microstructural features of CG scaffolds [17,20]. This modeling is possible because the pore structure of a variety of low-density, open-cell foams has been observed to have three consistent features [20]: an average of 14 faces per unit cell, 5.1 edges per face, and vertices that are nearly tetrahedral. These morphological features are observed as a result of a minimization of the total surface area of each unit cell. The tetrakaidecahedron is a polyhedron that packs to fill space, approximates the structural features of many experimentally characterized lowdensity, open-cell foams, nearly satisfies the minimum surface energy condition, and is often used for modeling such foams [21,22]. In addition, the value of the dimensionless measure of total edge length per (unit volume $)^{1 / 3}$ for the tetrakaidecahedral unit cell is nearly identical to that observed for many random cellular structures [23], suggesting that the tetrakaidecahedral unit cell gives a good representation of the total edge length and can be used to model the specific surface area of random cellular structures such as the porous, collagen-GAG scaffold.

A quantitative, cellular solids model describing the permeability $(k)$ of, or fluid mobility $(K)$ through, CG scaffolds in terms of scaffold mean pore size $(d)$, individual strut length $(l)$, percent compression $(\varepsilon)$, a dimensionless system constant $\left(A^{\prime}\right)$, and scaffold relative density $\left(\rho^{*} / \rho_{s}\right)$ has been developed from a series of known cellular solids relationships. The permeability $(k)$ of many open-cell foams has previously been reported in terms of mean pore size $(d)$ and relative density $\left(\rho^{*} / \rho_{s}, 1-\%\right.$ porosity) [20, 24]:

$$
k=A^{\prime} \cdot d^{2} \cdot\left(1-\frac{\rho^{*}}{\rho_{s}}\right)^{\frac{3}{2}}
$$

This model can be modified to describe scaffold fluid mobility $(K)$ using a single, system constant $\left(A^{\prime \prime}\right.$, $A^{\prime \prime}=A^{\prime} / \mu$ ) with dimensions $(\mathrm{Pa} \cdot \mathrm{s})^{-1}$ :

$$
K=\frac{A^{\prime}}{\mu} \cdot d^{2} \cdot\left(1-\frac{\rho^{*}}{\rho_{s}}\right)^{\frac{3}{2}}=A^{\prime \prime} \cdot d^{2} \cdot\left(1-\frac{\rho^{*}}{\rho_{s}}\right)^{\frac{3}{2}}
$$

The mean pore size of the compressed scaffold $\left(d_{\text {compress }}\right)$ under a given applied compressive strain $\left(\varepsilon_{a p p}\right)$ can be defined in terms of the initial edge length of the unit cell used to describe the scaffold microstructure $(l)$ [25]. A relationship between applied compressive strain and compressed mean pore size, modified from that proposed by Gent and Rusch [25] for this CG scaffold system with an observed Poisson's ratio of 0 , is presented here:

$$
d_{\text {compress }} \propto l \cdot\left(1-\varepsilon_{\text {app }}\right)
$$

Additionally, for the tetrakaidecahedral unit cell, scaffold mean pore size $(d)$ can be related to the average edge length of an individual strut $(l)$ within the unit cell [20]:

$$
d=2.785 \cdot l
$$

Combining these relationships, a single model describing scaffold permeability $(k)$ or fluid mobility $(K)$ in terms of a system constant $\left(A^{\prime}\right.$ or $\left.A^{\prime \prime}\right)$, scaffold mean pore size $(d)$, applied compressive strain $\left(\varepsilon_{\text {app }}\right)$, and relative density $\left(\rho^{*} / \rho_{s}\right)$ that is valid for any low-density, open-cell foam is proposed. These models are identical with the exception of the single, system constant $\left(A^{\prime \prime}=A^{\prime} / \mu\right)$ :

$$
k=A^{\prime} \cdot\left(\frac{d}{2.785}\right)^{2} \cdot(1-\varepsilon)^{2} \cdot\left(1-\frac{\rho^{*}}{\rho_{s}}\right)^{\frac{3}{2}}
$$




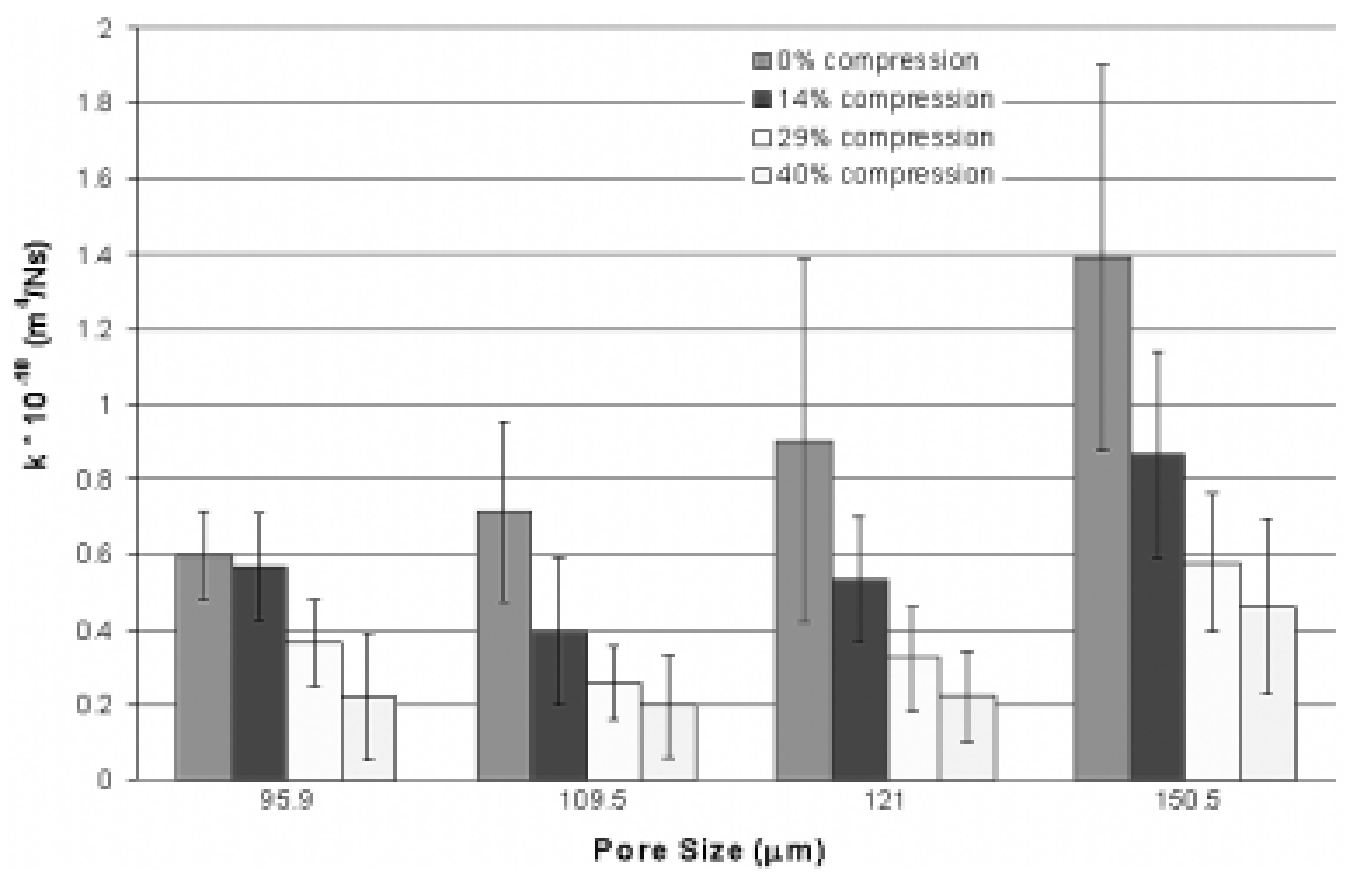

Fig. 2. Experimentally measured scaffold permeability (fluid mobility) as a function of pore size and applied compressive strain. One-way ANOVA tests revealed that mobility significantly increased with pore size and decreased with compressive strain.

$$
K=A^{\prime \prime} \cdot\left(\frac{d}{2.785}\right)^{2} \cdot(1-\varepsilon)^{2} \cdot\left(1-\frac{\rho^{*}}{\rho_{s}}\right)^{\frac{3}{2}}
$$

\subsection{Confocal microscopy}

The four scaffold variants with different mean pore sizes were each observed using confocal refection microscopy to acquire three-dimensional microstructural information and to investigate how different pore sizes might influence permeability. Circular specimens ( $8 \mathrm{~mm}$ diameter) were cut from each scaffold sheet using a dermal punch (Miltex, York, PA, USA). Each sample was then hydrated in saline solution for at least 24 hours prior to imaging. Confocal refection microscopy techniques utilizing an inverted Zeiss LSM 510 META (Gottingen, Germany) equipped with a 40x 1.3NA oil lens was then performed. A $488 \mathrm{~nm}$ (blue) Argon laser was used to illuminate the sample and a z-step of $1 \mu \mathrm{m}$ between images was used to optically section the samples to a depth of between 50 and $150 \mu \mathrm{m}$. The depth of imaging was influenced by the density of each scaffold section; in general, images were gathered until the signal lost resolution, which was on average approximately $100 \mu \mathrm{m}$ into the sample. The serial images were then reconstructed into a three-dimensional image using Zeiss image rendering software.

\section{Results}

The device designed and constructed to measure scaffold permeability proved to be effective for all scaffold variants and for all levels of applied compressive strain. CG scaffold permeability (fluid mobility) was found to be on the order of $10^{-10} \mathrm{~m}^{4} / \mathrm{Ns}$. While expected since the scaffold in the dry state 


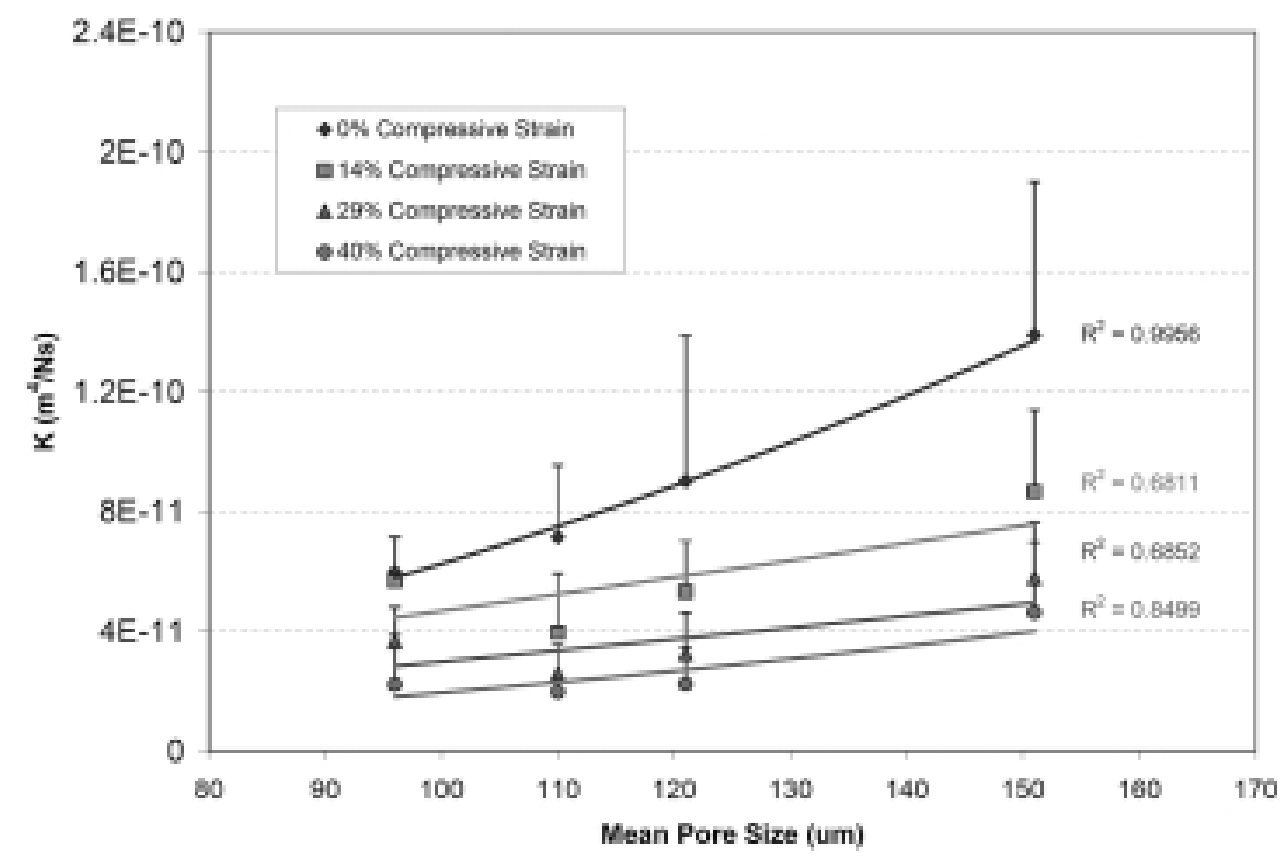

Fig. 3. Power regression between scaffold permeability (fluid mobility) (K) and mean pore size (96-151 $\mu \mathrm{m}$ ) for all four levels of applied compressive strain (0-40\%), testing the relationship proposed by the cellular solids model: $K \alpha d^{2}$. A good fit was found for all four levels of applied strain.

has a porosity of $99.5 \%$, this is quite high when compared to tissues such as cartilage $\left(10^{-15} \mathrm{~m}^{4} / \mathrm{Ns}\right)$ but several orders lower than that of trabecular bone $\left(10^{-6} \mathrm{~m}^{4} / \mathrm{Ns}\right)$. Figure 2 shows the experimentally measured scaffold permeability values (mean $\pm \mathrm{StDev}$ ) as a function of pore size and compressive strain. One-way ANOVA tests revealed that scaffold permeability increased significantly with pore size: $K=$ $0.660 \pm 0.256 \mathrm{~m}^{4} / \mathrm{Ns}$ to $K=1.387 \pm 0.516 \times 10^{-10} \mathrm{~m}^{4} / \mathrm{Ns}$ for the 96 and $151 \mu \mathrm{m}$ pore size scaffolds at $0 \%$ compression, respectively. Scaffold permeability was also observed to decrease with increasing compressive strain; $K=0.660 \pm 0.256 \times 10^{-10} \mathrm{~m}^{4} / \mathrm{Ns}$ and $0.231 \pm 0.180 \times 10^{-10} \mathrm{~m}^{4} / \mathrm{Ns}$ for the $96 \mu \mathrm{m}$ pore size at 0 and $40 \%$ compressive strain, respectively. Similar trends were found for the other scaffold variants (Fig. 2).

Power law regression analyses was performed between measured scaffold permeability (fluid mobility) $(K)$ and both the mean pore size $(d)$ and applied compressive strain $(\varepsilon)$. The first regression (Fig. 3) indicated a good correlation between $K$ and $d^{2}$ for $0 \%\left(\mathrm{R}^{2}=0.9956\right), 14 \%\left(\mathrm{R}^{2}=0.6811\right), 29 \%\left(\mathrm{R}^{2}=\right.$ $0.6852)$, and $40 \%\left(\mathrm{R}^{2}=0.8499\right)$ compressive strain, in agreement with the relationship between $K$ and $d$ proposed by the cellular solids model. In addition, a power law regression was performed between scaffold permeability $(K)$ and $(l-\varepsilon)$, where $(l-\varepsilon)$ corresponds to the remaining relative thickness of the compressed sample (Fig. 4). A good correlation was observed between $K$ and $(1-\varepsilon)^{2}$ for CG scaffolds with mean pore sizes of $96 \mu \mathrm{m}\left(\mathrm{R}^{2}=0.8911\right), 110 \mu \mathrm{m}\left(\mathrm{R}^{2}=0.9498\right), 121 \mu \mathrm{m}\left(\mathrm{R}^{2}=0.9773\right)$, and 151 $\mu \mathrm{m}\left(\mathrm{R}^{2}=0.9691\right)$, again in agreement with the relationship proposed by the cellular solids model.

The mathematical modeling showed remarkable accuracy in predicting the permeability of the constructs for all pore sizes and at each level of compression in a single model. Table 3 and Fig. 5 shows a comparison between the measured experimental results $\left(K_{\text {meas }}\right)$ and the predicted values obtained from the mathematical model $\left(K_{\text {calc }}\right)$ for the four distinct scaffold variants at the four levels of compressive 
Table 2

Structural properties of the collagen-GAG scaffold in its dry state. Varying the final freezing temperature produces scaffolds with uniform porosity (solid volume fraction), but with varying pore size, and consequently, varying specific surface area [17]

\begin{tabular}{cccc}
\hline$T_{f}\left({ }^{\circ} \mathrm{C}\right)$ & Mean Pore Size $(\mu \mathrm{m})$ & Solid Volume Fraction & Specific Surface Area $\left(\mu \mathrm{m}^{-1}\right)$ \\
\hline-10 & $150.5 \pm 32.1$ & 0.0048 & 0.00477 \\
-20 & $121.0 \pm 22.5$ & 0.0051 & 0.00593 \\
-30 & $109.5 \pm 18.3$ & 0.0050 & 0.00655 \\
-40 & $95.9 \pm 12.3$ & 0.0048 & 0.00748 \\
\hline
\end{tabular}

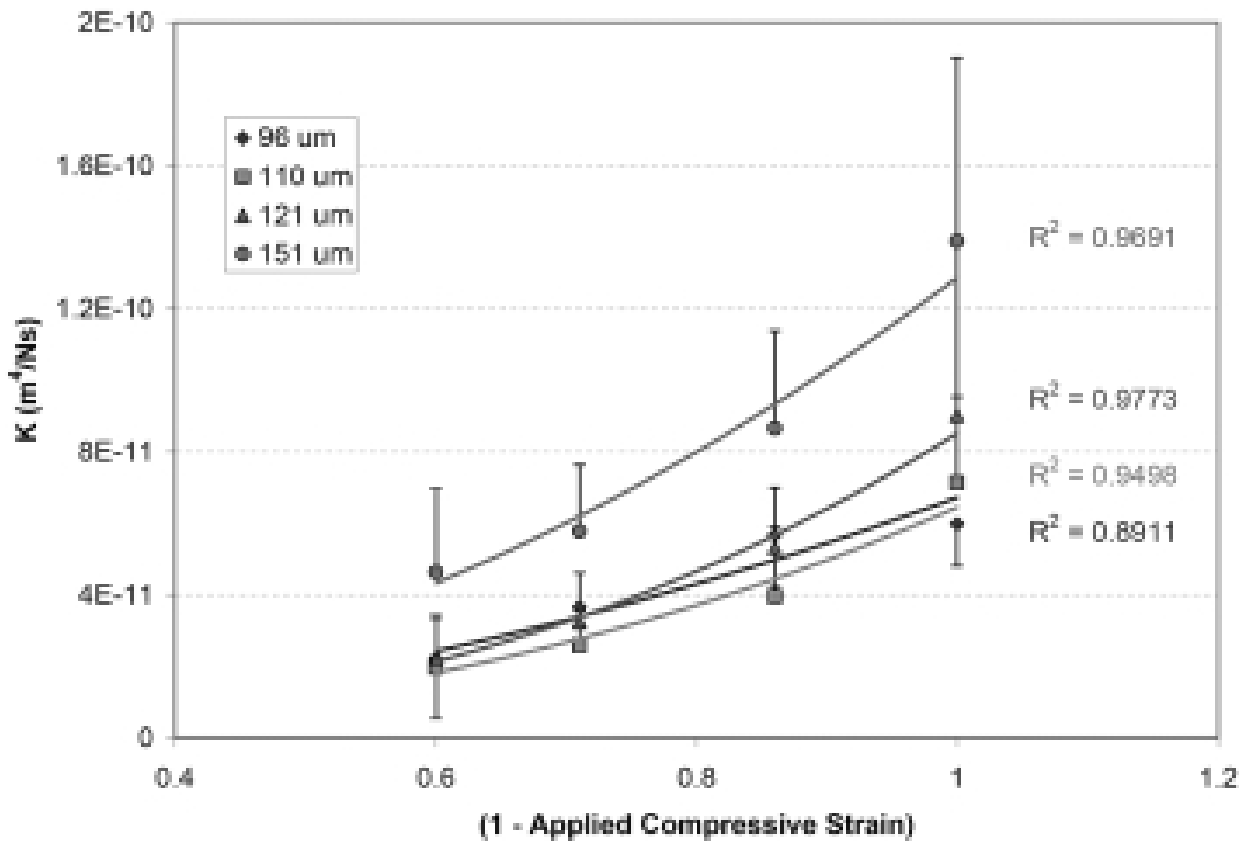

Fig. 4. Power regression between scaffold permeability (fluid mobility) (K) and applied compressive strain ( $\varepsilon$ : $0-40 \%)$ for all four scaffold variants (Mean pore size: $96-151 \mu \mathrm{m})$, testing the relationship proposed by the cellular solids model: $K \alpha(1-$ $\varepsilon)^{2}$. A good fit was found for all four scaffold variants.

strain. The cellular solids model predictions for all groups fit within the standard deviation of each experimentally measured value.

Figure 6 shows the confocal images taken of all four scaffold variants. The images reveal a complex and random microstructure in each of the scaffolds. When the images are compared, a clear relationship is evident between the pore size and the resulting surface area of the scaffolds. The images indicate that as the pore size increases, the specific surface area or the amount of collagen present in an average pore decreases. The scaffold with the largest pore size, $151 \mu \mathrm{m}$, has a distinct structure compared to the other variants. This scaffold has clearly a lower surface area than the other variants $(96,110$, or $121 \mu \mathrm{m}$ pore sizes).

\section{Discussion}

The device designed and constructed to experimentally measure permeability of collagen-GAG scaffolds for this investigation worked effectively. The device used in this study is similar to other devices 
Table 3

Experimentally measured $\left(K_{\text {meas }}\right.$, Mean \pm StDev) and cellular solids model derived $\left(K_{\text {calc }}\right)$ fluid mobility of the four CG scaffold variants (Mean Pore Size $=96,110,121,151 \mu \mathrm{m})$ under four levels of compressive strain $(\varepsilon=0,14,29$, $40 \%$ ). Units for $\mathrm{K}$ are $1 \times 10^{-10} \mathrm{~m}^{4} / \mathrm{Ns}$

\begin{tabular}{|c|c|c|c|c|c|c|c|c|}
\hline $\begin{array}{c}\text { Mean Pore } \\
\text { Size, } \mu \mathrm{m}\end{array}$ & $\begin{array}{l}\mathrm{K}_{\text {meas }} \\
\quad \varepsilon=0 \%\end{array}$ & $\mathrm{~K}_{\text {calc }}$ & $\begin{array}{l}\mathrm{K}_{\text {meas }} \\
\quad \varepsilon=14 \%\end{array}$ & $\mathrm{~K}_{\text {calc }}$ & $\begin{array}{l}\mathrm{K}_{\text {meas }} \\
\quad \varepsilon=29 \%\end{array}$ & $\mathrm{~K}_{\text {calc }}$ & $\begin{array}{l}\mathrm{K}_{\text {meas }} \\
\quad \varepsilon=40 \%\end{array}$ & $\mathrm{~K}_{\text {calc }}$ \\
\hline 96 & $0.598 \pm 0.117$ & 0.564 & $0.568 \pm 0.146$ & 0.417 & $0.365 \pm 0.114$ & 0.284 & $0.222 \pm 0.166$ & 0.203 \\
\hline 110 & $0.714 \pm 0.240$ & 0.735 & $0.396 \pm 0.196$ & 0.544 & $0.260 \pm 0.097$ & 0.371 & $0.197 \pm 0.136$ & 0.265 \\
\hline 121 & $0.902 \pm 0.483$ & 0.898 & $0.532 \pm 0.167$ & 0.664 & $0.324 \pm 0.137$ & 0.453 & $0.223 \pm 0.121$ & 0.323 \\
\hline 151 & $1.389 \pm 0.513$ & 1.389 & $0.866 \pm 0.273$ & 1.027 & $0.578 \pm 0.186$ & 0.700 & $0.463 \pm 0.233$ & 0.500 \\
\hline
\end{tabular}

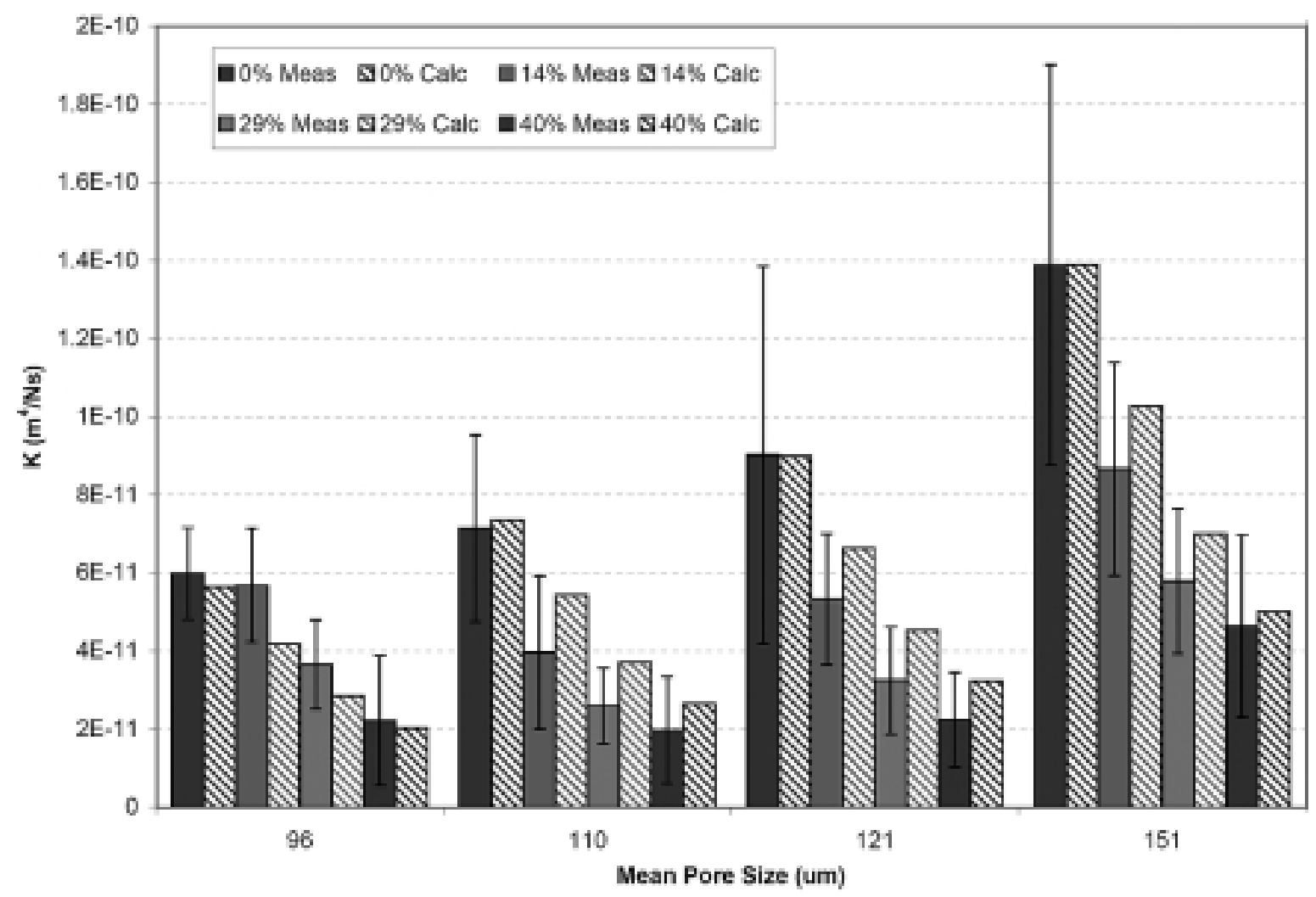

Fig. 5. Comparison between the experimental results $\left(K_{\text {meas }}\right)$ obtained and the predicted values obtained from the mathematical model $\left(K_{\text {calc }}\right)$ for CG scaffold permeability (fluid mobility) under varying compressive strains.

used to directly measure 3D construct permeability because it applies a constant pressure and measures the flow-rate through a material. The difference between this experimental device and others in the literature is the mechanism by which the material is secured. Typically, these devices have used adhesives or bonding cement to hold the material sample in place; this technique is effective for characterizing gel constructs and tissue samples. However such bonding techniques are not viable for highly-porous, cellular materials such as CG scaffolds that also exhibit viscoelastic behavior when hydrated. In the device developed for this study, the scaffold edges were clamped in place, resulting in a secure boundary between the sample and walls of the device; this arrangement also allowed various levels of compressive strain to be applied using spacers of varying thickness without severely distorting the scaffold. 

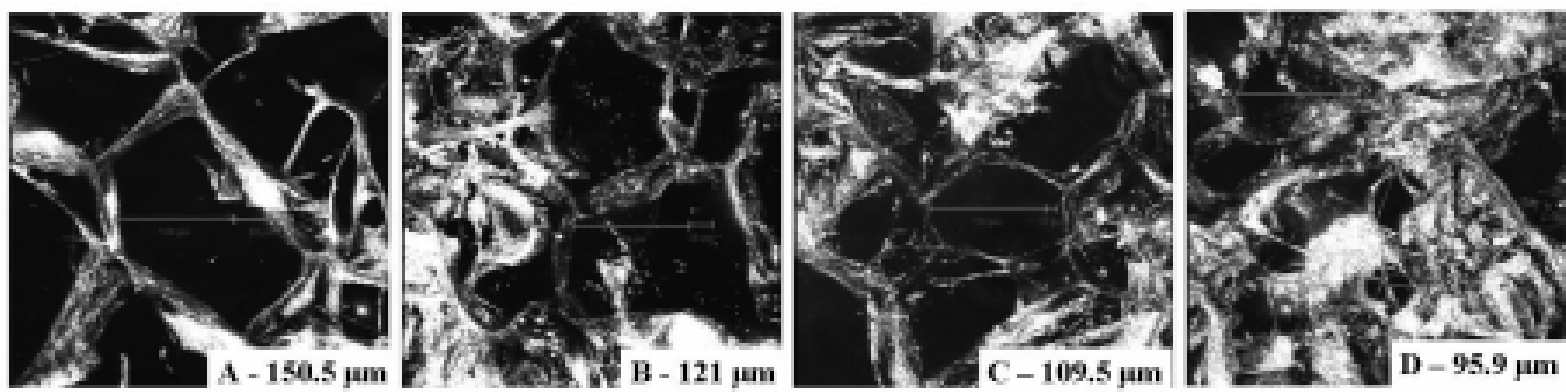

Fig. 6. Confocal refection images of each of the scaffold variants. Images suggest an increase in specific surface area with decreasing pore size. Scale bars are $150,120,110$, and $100 \mu \mathrm{m}$ for images A, B, C, and D, respectively.

The results from both the experimental and the cellular solids modelling analysis reveal that scaffold permeability/fluid mobility increased with increasing pore size and that scaffold specific surface area decreases with increasing mean pore size. The confocal refection microscopy analysis carried out in this study supported the hypothesis that increased specific surface area is found in scaffolds with smaller mean pore size. Furthermore, the permeability and specific surface area results are consistent with our hypothesis that increased scaffold permeability would be observed in scaffolds with increased mean pore size due to the corresponding reduction in scaffold specific surface area. These permeability results are also consistent with previous mathematical models describing the permeability of low porosity $(<90 \%)$ foams. The Carman-Kozeny mathematical model for foam permeability [12] described a number of geometric factors that influence the permeability of such foams: porosity, tortuosity (pore interconnectivity), pore size and orientation, fenestration size and shape, and specific surface area. The specific surface area influences permeability because it defines the total surface area within the scaffold; frictional effects that disturb and impede fluid flow resulting in reduced construct permeability are proportional to the scaffold surface area. While the Carman-Kozeny theoretical model has been successfully applied to a number of materials, the model is not applicable to highly porous materials (porosities greater than 90\%), such as the constructs used in this study and most tissue engineering scaffolds. The results from this study however, demonstrate that even in materials with a high porosity, scaffold mean pore size defines the scaffold specific surface area which in turn influences the ability of a fluid to pass through it. In addition, the particular relationship between scaffold permeability and mean pore size, $K \alpha d^{2}$, that is predicted using the cellular solids model is also observed experimentally, further validating the cellular solids model.

The results of the experimental and cellular solids modelling analysis also show that scaffold permeability decreases with increasing levels of compressive strain. This result is similar to the relationship between compression and permeability described by Lai and Mow [27] for articular cartilage. Other biological materials have also been found to follow this relationship, including the human medial collateral ligament [28]. The reason for this relationship in the collagen-GAG scaffolds is intuitive: as the material is compressed, the spacing between the collagen-GAG fibres decreases. Furthermore, the porosity of the constructs will also decrease under compression. Both of these factors would lead to the resultant increased resistance to fluid flow. In addition, the particular relationship between scaffold permeability and compressive strain, $k \alpha(1-\varepsilon)^{2}$, that is predicted using the cellular solids model is also observed experimentally, further validating the cellular solids model. The relationship between compressive strain and permeability in the collagen-GAG scaffolds will be useful in designing in vitro bioreactor-based culture protocols and for designing constructs for in vivo studies where significant loads may be applied 
to the scaffold. In a bioreactor we seek to apply the optimal biophysical stimuli to encourage cells to differentiate to specific phenotypes or to produce extracellular matrix formation. As the degree of compression to which a cell-seeded construct is exposed influences the permeability of fluid through the construct, the relationship between scaffold permeability with mean pore size, applied strain, and relative density will be used to determine the optimal bioreactor conditions required for specific tissue engineering applications. In addition, as constructs increase in size, it will be important to design appropriate microstructure in the scaffold to allow maximal nutrient influx to support scaffolds implanted into in vivo wound sites. Such utilizations of scaffold modeling tools and experimental measurements of scaffold permeability for designing the microstructure of future scaffolds is currently the subject of ongoing research in our laboratory.

The permeability/fluid mobility of the CG scaffolds was found to be in the order of $10^{-10} \mathrm{~m}^{4} / \mathrm{Ns}$. The only previous permeability value found in the literature for a collagen material was for a collagen gel [13] (Table 2). The values for permeability with these gels ranged from $1 \times 10^{-15}$ to $1 \times 10^{-16} \mathrm{~m}^{2}(1 \times$ $10^{-12} \mathrm{~m}^{4} / \mathrm{Ns}$ to $\left.1 \times 10^{-13} \mathrm{~m}^{4} / \mathrm{Ns}\right)$. The CG scaffold variants characterized in this study were $2-3$ orders of magnitude more permeable than these collagen gels, a result that makes intuitive sense due to the gel's significantly lower porosity. From a tissue engineering perspective, such differences in construct permeability suggest significantly higher levels of metabolite diffusion will be observed in a scaffold structure as compared to a gel, allowing increased cell proliferation and facilitating cell migration further into the scaffold.

The excellent comparison between experimentally measured and cellular solids model predicted scaffold permeability suggests that cellular solids modeling techniques can be used as a predictive model of scaffold permeability for many different scaffold architectures under a variety of physiological loading conditions, and as a predictive model to describe the permeability characteristics of future scaffold structures. Furthermore, it once again validates our use of low-density, open cell foam cellular solids modelling technique using a tetrakaidecahedral unit cell to model the random cellular structure of the collagen-GAG scaffolds. Previously we have shown that the same model can be used to predict the specific surface areas of the different scaffold variants [17]. Appropriate modeling tools that quantitatively describe scaffold characteristics (e.g. permeability, specific surface area, mechanical properties) in terms of salient microstructural features are advantageous for further advancement in the field of tissue engineering, and it appears that cellular solids modeling techniques utilizing the tetrakaidecahedral unit cell can accurately model CG scaffolds and have the potential to quantitatively describe many other tissue engineering constructs.

Confocal imaging (Fig. 6) allows visualization of the local three-dimensional microstructure of the four distinct scaffold variants and qualitative comparison of their relative specific surface areas. Qualitative analysis of these images suggest that as the pore size increases, the specific surface area decreases. Previously in our laboratory [17], we carried out an experiment where MC3T3-E1 mouse clonal osteogenic cells were seeded onto the four scaffold types used in this study, and maintained in culture for 24 and 48 hours. It was observed that scaffolds with different mean pore sizes had significantly different levels of cell attachment, in particular that as pore size decreased in the collagen-GAG scaffolds, the level of cell adhesion increased. We hypothesised that this effect might be due to a lower specific surface area in scaffolds with a larger mean pore size, resulting in a lower ligand density available for adhesion. The results of the confocal microscopy analyses of the same scaffold variants used in the cell attachment study performed here support this hypothesis.

In conclusion, we have demonstrated that that the permeability of collagen-GAG scaffolds decreases with decreasing pore size and with increasing compressive strain, have developed appropriate modelling 
tools to capture to the permeability behaviour of these scaffolds, and have observed the previously hypothesized relation between scaffold mean pore size and specific surface area. This data is currently being utilized during the development of fluid perfusion bioreactors for bone tissue engineering.

\section{Acknowledgements}

We are grateful for funding provided by a Science Foundation Ireland President of Ireland Young Researcher Award (FJOB), Cycle 3 of the PRTLI by the Higher Education Authority of Ireland (MAW) and the Cambridge-MIT Institute (BAH).

\section{References}

[1] M. Knothe-Tate and U. Knothe, An ex vivo model to study transport processes and fluid flow in loaded bone, J. Biomech. 33 (2000), 247-254.

[2] P.A. Netti, D.A. Berk, M.A. Swartz, A.J. Grodzinsky and R.K. Jain, Role of extracellular matrix assembly in interstitial transport in solid tumors, Can. Res. 60 (2000), 2497-2503.

[3] C.A. Znati, M. Rosenstein, T.D. McKee, E. Brown, D. Turner, W.D. Bloomer, S. Watkins, R.K. Jain and Y. Boucher, Irradiation reduces interstitial fluid transport and increases the collagen content in tumors, Clin. Can. Res. 9 (2003), $5508-5513$.

[4] J.M. Mansour and V.C. Mow, The permeability of articular cartilage under compressive strain and at high pressures, $J$. Bone Joint Surg. 58A(4) (1976), 509-516.

[5] A. Maroudas, P. Bullough, S.A. Swanson and M.A. Freeman, The permeability of articular cartilage, J. Bone Joint Surg. Br. 50 (1968), 166-177.

[6] A. Maroudas, Physiochemical properties of cartilage in the light of ion exchange theory, Biophy. 8 (1968), 575-595.

[7] I. Owan, D.B. Burr, C.H. Turner, J. Qiu, Y. Tu and J.E. Onyia, Mechanotransduction in bone: osteoblasts are more responsive to fluid forces than mechanical strain, Am. J. Physiol. 273(3) (1997), C810-C815.

[8] M.V. Hillsley and J.A. Frangos, Bone tissue engineering: the role of interstitial fluid flow, Biotech. Bioeng. 43 (1994), 573-581.

[9] P.J. Prendergast, R. Huiskes and K. Soballe, Biophysical stimuli on cells during tissue differentiation at implant interfaces, J. Biomech. 30(6) (1997), 539-548.

[10] C.M. Agrawal, J.S. McKinney, D. Lanctot and K.A Athanasiou, Effect of fluid flow on the in vitro degradation kinetics of biodegradable scaffolds for tissue engineering, Biomat. 21 (2000), 2443-2452.

[11] L.I. Shihong, J.R.D. Wijn, L.I. Jiaping, P. Layrolle and K. De Groot, Macroporous biphasic calcium phosphate scaffold with high permeability/porosity ratio, Tiss. Eng. 9(3) (2003), 535-548.

[12] J.R. Levick, Flow through interstitium and other fibrous matrices, Quar. J. Exper. Physiol. 72 (1987), 409-438.

[13] S. Ramanujan, A. Pluen, T.D. McKee, E.B. Brown, Y. Boucher and R.K. Jain, Diffusion and convection in collagen gels: implications for transport in the tumor interstitium, Biophys. 83 (2002), 1650-1660.

[14] C.A. Grattoni, H.H. Al-Sharji, C. Yang, A.H. Muggeridge and R.W. Zimmerman, Rheology and permeability of crosslinked polyacrylamide gel, J. Coll. Inter. Sci. 240 (2001), 601-607.

[15] M.W. Beatty, A.K. Ojha, J.L. Cook, L.R. Alberts, G.K. Mahanna, L.R. Iwasaki and J.C. Nickel, Small intestinal submucosa versus salt-extracted polyglycolic acid-poly-l-lactic acid: a comparison of neocartilage formed in two scaffold materials, Tiss. Eng. 8(6) (2002), 955-968.

[16] T.L. Spain, C.M. Agrawal and K.A. Athanasiou, New technique to extend the useful life of a biodegradable implant, Tiss. Eng. 4(4) (1998), 343-352.

[17] F.J. O’Brien, B.A. Harley, I.V. Yannas and L.J. Gibson, Effect of pore size on cell adhesion in collagen-gag scaffolds, Biomat. 26(4) (2005), 433-441.

[18] F.J. O'Brien, B.A. Harley, I.V. Yannas and L.J. Gibson, Influence of freezing rate on pore structure in freeze-dried collagen-gag scaffolds, Biomat. 25(6) (2005), 1077-1086.

[19] I.V. Yannas, E. Lee, D.P. Orgill, E.M. Skrabut and G.F. Murphy, Synthesis and characterization of a model extracellular matrix that induces partial regeneration of adult mammalian skin, Proc. Natl. Acad. Sci. 86 (1989), 933-937.

[20] L.J. Gibson and M.F. Ashby, Cellular Solids: Structure and Properties, in: Cambridge Solid State Science Series, D.R. Clarke, S. Suresh and I.M. Ward, eds, Cambridge University Press: Cambridge, UK, 1997.

[21] R.E. Williams, Space-filling polyhedron: its relation to aggregates of soap bubbles, plant cells and metal crystallites, Science 161 (1968), 276-277. 
[22] W. Thompson, On the division of space with minimum partition energy. Phil. Mag. 24, 1887503.

[23] A.M. Kraynik, D.A. Reinelt and F. van Swol, Structure of random monodisperse foam, Phys. Rev. 67 (2003), Art. No. 031403 Part 1.

[24] W.F. Brace, Permeability from resistivity and pore shape, J. Geophys. Res. 82 (1977), 3343-3349.

[25] A.N. Gent and K.C. Rusch, Permeability of open-cell foamed materials, J. Cell. Plast. 2 (1966), 46-51.

[26] S.M. Haddock, J.C. Debes, E.A. Nauman, K.E. Fong, Y.P Arramon and T.M. Keaveny, Structure-function relationships for coralline hydroxyapatite bone substitute, J. Biomed. Mater. Res. 47 (1999), 71-78.

[27] W.M. Lai and V.C. Mow, Drag-induced compression of articular cartilage during a permeation experiment, Biorheol. 17 (1980), 111-123.

[28] E.A. Sander and E.A Nauman, Permeability of musculoskeletal tissues and scaffolding materials: experimental results and theoretical predictions, Crit. Rev. Biomed. Eng. 31(1) (2003), 1-26. 